\title{
ESTUDO POR ESPECTROSCOPIA MICRO-RAMAN DOS MECANISMOS DE SEPARAÇÃO DE FASE EM VIDROS FOSFATOS DE METAIS DE TRANSIÇÃO
}

\author{
Italo Odone Mazali* e Oswaldo Luiz Alves \\ Instituto de Química, Universidade Estadual de Campinas, CP 6154, 13083-970 Campinas - SP, Brasil \\ Iara de Fátima Gimenez \\ Departamento de Química, Universidade Federal de Sergipe, 49100-000 São Cristóvão - SE, Brasil
}

Recebido em 17/10/08; aceito em 12/3/09; publicado na web em 10/8/09

\begin{abstract}
MICRO-RAMAN SPECTROSCOPY STUDIES OF THE PHASE SEPARATION MECHANISMS OF TRANSITION-METAL PHOSPHATE GLASSES. Glass-ceramics are prepared by controlled separation of crystal phases in glasses, leading to uniform and dense grain structures. On the other hand, chemical leaching of soluble crystal phases yields porous glass-ceramics with important applications. Here, glass/ceramic interfaces of niobo-, vanado- and titano-phosphate glasses were studied by micro-Raman spectroscopy, whose spatial resolution revealed the multiphase structures. Phase-separation mechanisms were also determined by this technique, revealing that interface composition remained unchanged as the crystallization front advanced for niobo- and vanadophosphate glasses (interface-controlled crystallization). For titanophosphate glasses, phase composition changed continuously with time up to the equilibrium composition, indicating a spinodal-type phase separation.
\end{abstract}

Keywords: Raman spectroscopy; crystallization; phosphate glasses.

\section{INTRODUÇÃO}

Vidro é um sólido não-cristalino, portanto, com ausência de simetria e periodicidade translacional, que exibe o fenômeno de transição vítrea, podendo ser obtido a partir de qualquer material inorgânico, orgânico ou metálico e formado através de qualquer técnica de preparação. ${ }^{1} \mathrm{O}$ comportamento de transformação de fase em vidros, ou seja, sua transformação de sólido com ausência de periodicidade para um sólido estruturalmente organizado tem grande importância científica e tecnológica, pois, pode conduzir à obtenção de materiais cerâmicos avançados com alto valor agregado e funções específicas.

Materiais cerâmicos funcionais avançados preparados via transformação de fase de um vidro precursor oferecem vantagens sobre os métodos convencionais de sinterização de pós, pois, permitem o crescimento controlado dos cristais, com estrutura de grãos uniforme e livre de porosidade, além de evitar as variáveis intrínsecas do processo de sinterização.

A espectroscopia micro-Raman tem sido empregada de maneira sistemática no estudo de materiais vítreos, cuja caracterização por métodos de difração de raios X é prejudicada pela ausência de periodicidade a médias e longas distâncias. Na microscopia Raman ou espectroscopia micro-Raman, o espectrômetro Raman está acoplado a um microscópio óptico. ${ }^{2-4}$ Neste caso, o feixe de excitação é direcionado ao microscópio, que permite que a amostra seja examinada com base nas ampliações permitidas pela ótica do microscópio, propiciando que áreas específicas de até $1 \mu \mathrm{m}$ de diâmetro sejam analisadas seletivamente. ${ }^{4}$ Os feixes refletidos e espalhados, elástica e inelasticamente, são coletados através da objetiva do microscópio e direcionados ao espectrômetro, no qual o espalhamento Rayleigh deve ser separado por filtros holográficos do tipo notch. Para uma revisão detalhada dos fundamentos da espectroscopia vibracional e do espalhamento Raman, recomenda-se a leitura de uma série de trabalhos publicados recentemente por Sala. ${ }^{5-7}$

Com o notável desenvolvimento da instrumentação, a espectroscopia micro-Raman adquiriu uma grande versatilidade de aplicações,

\footnotetext{
*e-mail: mazali@iqm.unicamp.br
}

de tal forma que atualmente aplica-se a amostras biológicas, ${ }^{8,9}$ peças de arte,,$^{10}$ amostras de origem arqueológica ${ }^{4,11-13}$ tais como vidros, cerâmicas, tecidos históricos, manuscritos e pinturas rupestres. A espectroscopia micro-Raman é apontada como uma das principais técnicas que, usadas isoladamente, permitem o estudo de misturas heterogêneas de sólidos orgânicos ou inorgânicos, sendo particularmente adequadas ao estudo de sistemas multifásicos e multicomponente, mesmo em escala nanométrica. ${ }^{14}$ Algumas vantagens da espectroscopia micro-Raman merecem destaque: ${ }^{14}$ trata-se de uma técnica não-destrutiva e que pode ser aplicada a amostras de formas irregulares e tamanhos variados; apresenta uma alta resolução espacial e espectral; permite a análise de amostras "reais", sem etapas de preparação prévia; em alguns casos, o instrumento pode apresentar portabilidade, ou seja, pode ser transportado a campo.

A evolução estrutural envolvida na transformação de um vidro em cerâmica, via tratamentos térmicos, pode ser acompanhada pela microscopia Raman, evidenciando detalhadamente a transformação de fase. ${ }^{15-17}$ A separação de fases cristalinas em vidros, induzida por irradiação com lasers, é outra linha bastante representativa do uso da espectroscopia micro-Raman ao estudo de sistemas vítreos multifásicos. ${ }^{18-20} \mathrm{~A}$ grande vantagem neste último caso é a formação das fases cristalinas restrita à superfície dos vidros, com morfologia controlada, que pode ser caracterizada com precisão com a espectroscopia microRaman. Por outro lado, a determinação dos mecanismos de separação de fases em vidros é uma possibilidade ainda inexplorada, diante do grande potencial da técnica. Em virtude disto, este trabalho descreve o uso da espectroscopia micro-Raman para a determinação do mecanismo de separação de fases cristalinas em vidros fosfatos.

Os processos de transformação de fase em vidros podem ser divididos em dois grupos principais: $:^{21,22}$ (a) decomposição espinoidal (separação de fase líquido-líquido): neste processo, uma fase simples homogênea pode separar-se em duas ou mais fases de composições diferentes, se a energia livre do sistema com duas ou mais fases distintas for menor do que aquela do sistema com uma fase simples homogênea. Na decomposição espinoidal uma mudança contínua da composição ocorre enquanto a situação de equilíbrio é atingida. A interface é inicialmente muito difusa, mas define-se gradualmente e 
a segunda fase é distribuída regularmente havendo uma tendência de separar-se com uma estrutura não esférica com grande conectividade; b) cristalização: a cristalização de vidros é controlada basicamente por dois fatores. O primeiro é a taxa de nucleação, isto é, o número de núcleos formados por unidade de volume por unidade de tempo; o segundo é a taxa de crescimento de cristais. Se a cristalização ocorre por núcleos dispersos por toda a matriz do vidro, esta é denominada cristalização de volume. Três tipos básicos de nucleação podem ocorrer durante a cristalização de volume: nucleação homogênea: ocorre arbitrariamente por todo o sistema sem preferência por sítios para a formação dos núcleos; nucleação heterogênea: envolve a formação de núcleos de uma nova fase na superfície de contorno de uma fase já existente. Se a nucleação ocorre no contorno de fase, por exemplo, entre a superfície do vidro e o ar, então a cristalização prossegue desta superfície em direção ao centro da matriz, sendo então denominada de cristalização de superfície; fotonucleação: a presença de pequenas quantidades de íons metálicos, tais como, cobre, ouro ou prata, sob efeito da radiação ultravioleta e tratamento térmico, induz a formação de cristalitos coloidais destes metais nas áreas irradiadas, os quais atuam como agentes de nucleação, promovendo a cristalização do vidro. A Tabela 1 traz uma comparação entre os dois principais processos de transformação de fase em vidros.

Tabela 1. Comparação entre a transformação de fase em vidros por decomposição espinoidal e por cristalização (nucleação e crescimento)

\begin{tabular}{|c|c|}
\hline Nucleação e crescimento & Decomposição espinoidal \\
\hline $\begin{array}{l}\text { A composição da fase nucleada } \\
\text { permanece constante com o } \\
\text { tempo. } \\
\text { A interface entre as fases é } \\
\text { quase sempre bem definida } \\
\text { durante o crescimento. } \\
\text { Apresenta uma tendência da } \\
\text { fase separar como esferas com } \\
\text { pequena conectividade. }\end{array}$ & $\begin{array}{l}\text { Ocorre uma mudança contínua da } \\
\text { composição enquanto a situação } \\
\text { de equilíbrio é atingida. } \\
\text { Inicialmente a interface é muito } \\
\text { difusa, mas define-se gradual- } \\
\text { mente. } \\
\text { Apresenta uma tendência da fase } \\
\text { separar com uma estrutura não- } \\
\text { esférica com grande conectivi- } \\
\text { dade. }\end{array}$ \\
\hline
\end{tabular}

O comportamento de um vidro frente à separação de fases cristalinas pode ser estudado por meio de métodos cinéticos baseados, pelo menos em parte, no formalismo desenvolvido por Avrami para reações de estado sólido, utilizando-se dados provenientes de curvas de análise térmica (DTA, DSC). ${ }^{23}$

O objetivo deste trabalho é demonstrar a potencialidade da espectroscopia micro-Raman na identificação dos processos de transformação de fase em vidros fosfatos com base nas características de cada processo exposto na Tabela 1, bem como a identificação dos estágios sequenciais de separação de cada fase cristalina. As potencialidades da espectroscopia micro-Raman foram evidenciadas a partir do estudo da transformação de fase dos vidros $6 \mathrm{Li}_{2} \mathrm{O}-24 \mathrm{TiO}_{2}-39 \mathrm{CaO}-31 \mathrm{P}_{2} \mathrm{O}_{5}$ (LTCP) ${ }^{17,24,25}, 6 \mathrm{Li}_{2} \mathrm{O}-18 \mathrm{Nb}_{2} \mathrm{O}_{5}-43 \mathrm{CaO}-33 \mathrm{P}_{2} \mathrm{O}_{5}(\mathrm{LNCP})^{16}$ e $6 \mathrm{Li}_{2} \mathrm{O}-$ $18 \mathrm{~V}_{2} \mathrm{O}_{3}-43 \mathrm{CaO}-33 \mathrm{P}_{2} \mathrm{O}_{5}(\mathrm{LVCP}) .{ }^{15}$

\section{METODOLOGIA}

Os vidros LTCP, LNCP e LVCP foram preparados fundindo-se os reagentes, na forma dos seus óxidos ( $\left.\mathrm{TiO}_{2}, \mathrm{Nb}_{2} \mathrm{O}_{5}, \mathrm{~V}_{2} \mathrm{O}_{3}, \mathrm{P}_{2} \mathrm{O}_{5}\right)$ e carbonatos $\left(\mathrm{Li}_{2} \mathrm{CO}_{3}\right.$ e $\left.\mathrm{CaCO}_{3}\right)$, em cadinho de platina, a $1350{ }^{\circ} \mathrm{C}$ por $1 \mathrm{~h}$, utilizando um forno marca EDG, modelo F1700, em atmosfera ambiente. As misturas foram preparadas calculando-se as porcentagens em mol (mol\%) dos reagentes, na forma de seus respectivos óxidos, de modo a se obter uma massa final de vidro igual a $25 \mathrm{~g}$. Durante a fusão, o cadinho com o fundido foi agitado várias vezes, para assegurar a homogeneização do fundido. Após o período de refino, o fundido foi retirado rapidamente do forno e vazado sobre molde de grafite e, após alguns segundos, já tendo adquirido rigidez, foi submetido a recozimento nas temperaturas: $\mathrm{LTCP}=630^{\circ} \mathrm{C}, \mathrm{LNCP}$ $=550^{\circ} \mathrm{C}$ e $\mathrm{LVCP}=530^{\circ} \mathrm{C}$, por $2 \mathrm{~h}$, sob atmosfera ambiente.

Para o estudo do mecanismo de devitrificação, discos de $25 \mathrm{~mm}$ de diâmetro e $2 \mathrm{~mm}$ de espessura foram submetidos a um patamar de nucleação nas temperaturas: $\mathrm{LTCP}=630{ }^{\circ} \mathrm{C}, \mathrm{LNCP}=630{ }^{\circ} \mathrm{C} \mathrm{e}$ $\mathrm{LVCP}=530{ }^{\circ} \mathrm{C}$, por $20 \mathrm{~h}$. Após resfriamento, as amostras dos diferentes sistemas vítreos foram introduzidas no forno já aquecido na temperatura de devitrificação, $\mathrm{T}_{\mathrm{c}}$ : LTCP $=720^{\circ} \mathrm{C}, \mathrm{LNCP}=830^{\circ} \mathrm{C}$ e $\mathrm{LVCP}=600{ }^{\circ} \mathrm{C}$, em intervalos de tempo de 2 a 30 min e por $12 \mathrm{~h}$. Os valores de $\mathrm{T}_{\mathrm{c}}$ foram obtidos a partir das curvas DTA.

Os espectros micro-Raman foram obtidos no equipamento Renishaw Raman Imaging Microprobe System 3000, acoplado a um microscópio óptico com resolução espacial de $1,0 \mu \mathrm{m}$, que permite selecionar a região de interesse da amostra para a obtenção do espectro Raman, laser de $\mathrm{He}-\mathrm{Ne}(\lambda=632,8 \mathrm{~nm})$ com uma potência de $8 \mathrm{~mW}$ e resolução de $2 \mathrm{~cm}^{-1}$. As regiões analisadas foram selecionadas na superfície das amostras submetidas à devitrificação. Os pontos assinalados nas imagens de microscopia representam as regiões representativas investigadas e não o ponto exato analisado nas amostras.

\section{RESULTADOS E DISCUSSÃO}

A transformação de fase no vidro LTCP ocorre pelo processo de decomposição espinoidal. ${ }^{24,25}$ A Figura 1 mostra a transformação de fase do vidro LTCP, para diferentes tempos de tratamento térmico, onde podemos observar regiões brancas contínuas e difusas na matriz vítrea. A Figura 2 ilustra os espectros Raman das regiões assinaladas na Figura 1.

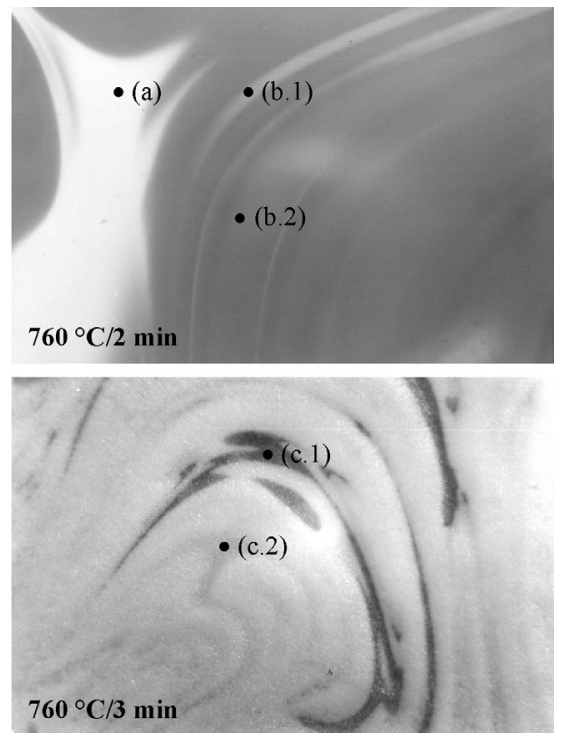

Figura 1. Micrografia óptica do início do processo de devitrificação do vidro LTCP submetido a tratamento térmico a $760{ }^{\circ} \mathrm{C} / 2 \mathrm{~min}$ e $760{ }^{\circ} \mathrm{C} / 3 \mathrm{~min}$ (aumento: $40 x$ )

Como descrito na Introdução, uma das características da decomposição espinoidal é a mudança contínua na composição enquanto a condição de equilíbrio é atingida. Para verificarmos esse efeito, submetemos o vidro LTCP a tratamento térmico a $760{ }^{\circ} \mathrm{C}$ por períodos de tempos que variaram entre 2 e $30 \mathrm{~min}$, além da condição de devitrificação adotada que foi de $760{ }^{\circ} \mathrm{C}$ por $12 \mathrm{~h}$. Durante os três 
primeiros minutos de tratamento, podemos observar duas regiões distintas na mesma peça devitrificada (Figura 1). As regiões denominadas como 1 , estão relacionadas às frações menos devitrificadas, visto que entre 800 e $1400 \mathrm{~cm}^{-1}$, os espectros Raman (Figura 2, b1 e c1) ainda apresentam um perfil semelhante ao vidro as cast (Figura 2a), caracterizado por uma banda intensa em $931 \mathrm{~cm}^{-1}$, relacionada à presença de grupos $\mathrm{TiO}_{6}$ na rede formadora do vidro. ${ }^{26} \mathrm{O}$ vidro LTCP é constituído de uma rede mista de titânio e fósforo ligados em ponte, prevalecendo uma cadeia tipo piro/ortofosfato, embora possa ser evidenciada também a presença de cadeias metafosfato remanescentes. ${ }^{27}$ Entretanto, a região entre 100 e $700 \mathrm{~cm}^{-1}$ já exibe bandas associadas às fases cristalinas formadas. As regiões denominadas como 2 (Figura 1) referem-se a frações com a devitrificação mais avançada, visto que os espectros Raman não exibem mais nenhum perfil semelhante ao do vidro (Figura 2, b2 e c2).

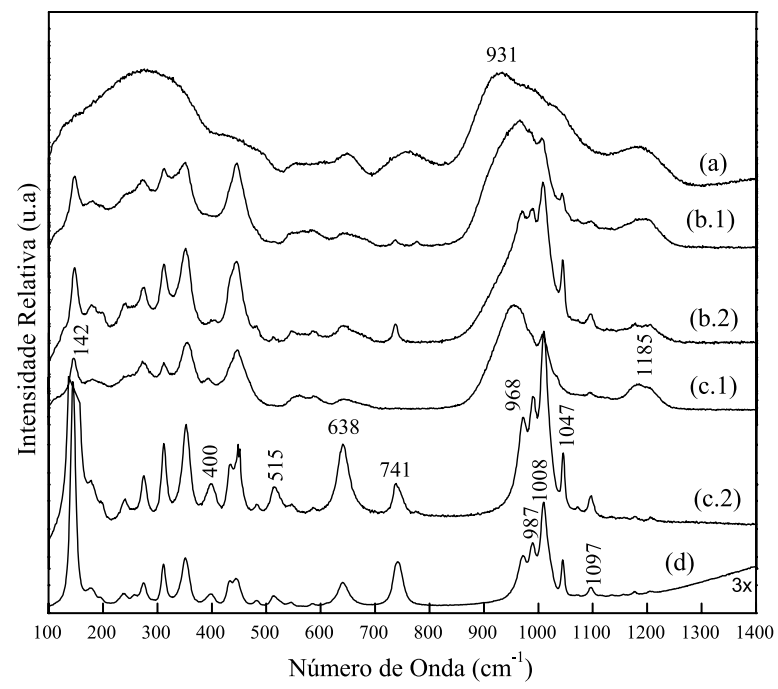

Figura 2. Espectros micro-Raman do vidro LTCP das diferentes regiões indicadas na Figura 1 e (d) submetido a tratamento térmico a $760{ }^{\circ} \mathrm{C} / 12 \mathrm{~h}$

Para todos os tempos de tratamento, as bandas na região entre $100 \mathrm{e} 700 \mathrm{~cm}^{-1}$ estão relacionadas à presença da fase $\mathrm{LiTi}_{2}\left(\mathrm{PO}_{4}\right)_{3} \cdot{ }^{28}$ A região do espectro entre 100 e $400 \mathrm{~cm}^{-1}$ está relacionada com os modos externos, translações e vibrações, as quais, em geral, só podem ser atribuídas por meio de um tratamento completo de fator grupo, o que não é o objetivo neste momento. De maneira geral, os compostos de fórmula $\mathrm{A}_{\mathrm{x}} \mathrm{B}_{2}\left(\mathrm{PO}_{4}\right)_{3}$, denominados NASICON, apresentam uma estrutura romboédrica $\mathrm{R} \overline{3} \mathrm{c}$, sobre a qual é feita a grande maioria dos tratamentos de fator grupo ${ }^{29}$ (onde $\mathrm{A}=$ metal alcalino, $\mathrm{B}=$ metais de transição, tais como $\mathrm{Ti}^{4+}, \mathrm{Zr}^{4+}, \mathrm{Nb}^{5+}, \mathrm{Ge}^{4+}$ e $\left.0<\mathrm{x} \leq 4\right)$ ). Porém, alguns compostos de lítio não são romboédricos, o que poderia dificultar ainda mais as atribuições.

Na região denominada 2, os espectros Raman já exibem o perfil da vitrocerâmica final, porém, podemos observar uma evolução no espectro com o tempo de tratamento térmico. Ao passarmos de 2 para 3 min a $760{ }^{\circ} \mathrm{C}($ b. $2 \rightarrow$ c. 2 , Figura 2$)$, ocorre um aumento na intensidade das bandas em 638,515 e $400 \mathrm{~cm}^{-1}$, além de uma grande intensificação na banda em $142 \mathrm{~cm}^{-1}$, as quais são atribuídas à fase $\mathrm{TiO}_{2}$ anatásio. ${ }^{30}$ Comparando-se as diferentes regiões 1 e 2 , para um mesmo tempo de tratamento térmico (b.1 $\Leftrightarrow$ b. 2 ou c. $1 \Leftrightarrow$ c. 2 , Figura 2 ), nota-se que essas regiões se diferem principalmente na região entre 850 e $1100 \mathrm{~cm}^{-1}$, passando de uma banda larga para uma banda com três ombros bem definidos em 968,987 e $1008 \mathrm{~cm}^{-1}$ associadas aos modos de estiramento simétrico e assimétrico da ligação P-O, na estrutura tipo NASICON.
Entre 5 min e $12 \mathrm{~h}$ de tratamento térmico, não se distinguem essas duas regiões e praticamente não ocorrem mais alterações nos espectros Raman (Figura 2d). A interpretação dos espectros Raman, com base na Figura 1, seguiu a seguinte sequência de evolução: vidro as cast (a), região b. $1\left(760^{\circ} \mathrm{C} / 2 \mathrm{~min}\right)$, região b. $2\left(760{ }^{\circ} \mathrm{C} / 2 \mathrm{~min}\right)$, região c. $2\left(760{ }^{\circ} \mathrm{C} / 3 \mathrm{~min}\right)$ e vitrocerâmica a $760{ }^{\circ} \mathrm{C} / 12 \mathrm{~h}$ (Figura $2 \mathrm{~d}$ ).

Em um primeiro momento, situação b.1 (Figura 2), teríamos apenas a separação de fase induzida pelos íons titânio presentes na rede como modificadores, uma vez que não verificamos alterações nas bandas entre 850 e $1100 \mathrm{~cm}^{-1}$, atribuídas ao modo P-O em uma rede tipo Ti-O-P. Na situação b.2 (Figura 2), temos o desdobramento da banda centrada em $931 \mathrm{~cm}^{-1}$ (relacionada à presença de grupos $\mathrm{TiO}_{6}$ na rede formadora do vidro LTCP) revelando que a estrutura vítrea dá lugar, gradualmente, aos arranjos ordenados típicos da fase cristalina $\mathrm{LiTi}_{2}\left(\mathrm{PO}_{4}\right)_{3}$. As fases tipo NASICON possuem tetraedros $\mathrm{PO}_{4}$, conectados a octaedros $\mathrm{TiO}_{6}$, através de pontes Ti-O-P nos vértices tendo, portanto, sua formação favorecida. Nesta situação, surgem também as bandas em 1047 e $741 \mathrm{~cm}^{-1}$, que são atribuídas, de forma inequívoca, à fase $\beta-\mathrm{Ca}_{2} \mathrm{P}_{2} \mathrm{O}_{7} \cdot{ }^{31} \mathrm{~A}$ formação dessa fase mostra-se favorável, uma vez que a formação de um ortofosfato, como o $\beta-\mathrm{Ca}_{3}\left(\mathrm{PO}_{4}\right)_{2}$, requer a quebra das ligações em ponte P-O-P e P-O-Ti das cadeias do vidro, ou mesmo, das cadeias NASICON. A ligação P-O-P, observada no vidro em $765 \mathrm{~cm}^{-1}$, se rearranjaria formando a fase $\beta-\mathrm{Ca}_{2} \mathrm{P}_{2} \mathrm{O}_{7}$. Entretanto, não pode ser desconsiderada a possibilidade de ocorrência da fase $\beta-\mathrm{Ca}_{3}\left(\mathrm{PO}_{4}\right)_{2}$, visto que suas bandas no espectro Raman são coincidentes com as demais bandas da fase $\beta-\mathrm{Ca}_{2} \mathrm{P}_{2} \mathrm{O}_{7}$.

Ao passarmos para a situação c.2 (Figura 2), verificamos uma melhor definição de todas as bandas, principalmente aquelas relacionadas à presença da fase $\mathrm{TiO}_{2}$ anatásio, indicando um processo de rearranjo das fases cristalinas. Observa-se, também, que a banda em $1185 \mathrm{~cm}^{-1}$, verificada nas situações a, b.1, b.2 e c.1 (Figura 2) e atribuída a cadeias metafosfato, não ocorre mais na situação c.2 (Figura 2). Este fato evidencia a fragmentação da cadeia do vidro, à medida que ocorre a transformação de fase via decomposição espinoidal.

Com relação à banda observada em $637 \mathrm{~cm}^{-1}$, que surge com forte intensidade na situação c.2 (Figura 2) e também se manifesta na vitrocerâmica final, esta foi atribuída à ocorrência da fase $\mathrm{Li}(\mathrm{TiO}) \mathrm{PO}_{4}{ }^{32}$ em detrimento da fase $\mathrm{TiO}_{2}$ anatásio, em decorrência da reação de estado sólido $\mathrm{LiTi}_{2}\left(\mathrm{PO}_{4}\right)_{3}+2 \beta-\mathrm{Ca}_{3}\left(\mathrm{PO}_{4}\right)_{2} \rightarrow \mathrm{Li}(\mathrm{TiO})$ $\mathrm{PO}_{4}+\mathrm{TiO}_{2}+3 \beta-\mathrm{Ca}_{2} \mathrm{P}_{2} \mathrm{O}_{7}$. Vale destacar que a fase $\beta-\mathrm{Ca}_{3}\left(\mathrm{PO}_{4}\right)_{2}$, estudada por Aza e colaboradores ${ }^{33}$ por espectroscopia Raman, não apresenta nenhuma banda no intervalo de 650 a $900 \mathrm{~cm}^{-1}$, o mesmo ocorrendo para a fase $\mathrm{LiTi}_{2}\left(\mathrm{PO}_{4}\right)_{3}$, que não apresenta nenhuma banda no intervalo de 550 a $900 \mathrm{~cm}^{-1}$. $^{28}$

A espectroscopia micro-Raman possibilitou a identificação das fases $\mathrm{LiTi}_{2}\left(\mathrm{PO}_{4}\right)_{3}, \beta-\mathrm{Ca}_{3}\left(\mathrm{PO}_{4}\right)_{2}, \mathrm{Li}(\mathrm{TiO}) \mathrm{PO}_{4}, \mathrm{TiO}_{2}, \beta-\mathrm{Ca}_{2} \mathrm{P}_{2} \mathrm{O}_{7}$ no processo de devitrificação do vidro LTCP. A partir desses resultados e mantendo a proporção estequiométrica entre os componentes do vidro e da vitrocerâmica, podemos propor uma equação para descrever o processo de devitrificação do vidro LTCP, onde x varia em função das condições do tratamento térmico:

$\left(6 \mathrm{Li}_{2} \mathrm{O}-24 \mathrm{TiO}_{2}-39 \mathrm{CaO}-31 \mathrm{P}_{2} \mathrm{O}_{5}\right)^{\text {vidro }} \rightarrow(12-\mathrm{x}) \mathrm{LiTi}_{2}\left(\mathrm{PO}_{4}\right)_{3}+$ $(13-2 \mathrm{x}) \beta-\mathrm{Ca}_{3}\left(\mathrm{PO}_{4}\right)_{2}+\mathrm{x} \mathrm{Li}(\mathrm{TiO}) \mathrm{PO}_{4}+3 \mathrm{x} \beta-\mathrm{Ca}_{2} \mathrm{P}_{2} \mathrm{O}_{7}+\mathrm{xTiO}_{2}$ (sendo $0 \leq \mathrm{x} \leq 6)$

Comparando-se os espectros Raman c. 2 e d, da Figura 2, podemos observar uma alteração na intensidade relativa das bandas em 638 e $741 \mathrm{~cm}^{-1}$. Como proposto pela Equação 1, o início do processo de devitrificação é caracterizado por $\mathrm{x}=0$ (situação representada pelo espectro c.2, da Figura 2). Podemos observar pela Equação 1, que para cada incremento de $\mathrm{x}$, temos a formação de $1 \mathrm{~mol}$ de $\mathrm{Li}(\mathrm{TiO}) \mathrm{PO}_{4}$ e 3 mols de $\beta-\mathrm{Ca}_{2} \mathrm{P}_{2} \mathrm{O}_{7}$. Consequentemente, com o avanço do processo 
de devitrificação, temos uma intensificação da banda em $741 \mathrm{~cm}^{-1} \mathrm{em}$ relação à banda em $638 \mathrm{~cm}^{-1}$ (representado pelo espectro d da Figura 2). Da mesma maneira, observamos uma intensificação da banda em $142 \mathrm{~cm}^{-1}$ (atribuída ao TiO anatásio) em comparação às bandas entre $968-1080 \mathrm{~cm}^{-1}$ atribuídas à fase $\mathrm{LiTi}_{2}\left(\mathrm{PO}_{4}\right)_{3}$. Tal argumento também se sustenta, ao observamos que ocorre uma intensificação da banda em $1047 \mathrm{~cm}^{-1}$ (atribuída ao $\beta-\mathrm{Ca}_{2} \mathrm{P}_{2} \mathrm{O}_{7}$ ) em relação às bandas da fase NASICON.

As Figuras 3 a e $3 b$ ilustram a evolução do processo de transformação de fase no vidro LNCP em função do tempo de tratamento térmico ( 2 e $5 \mathrm{~min}$, respectivamente, a $830^{\circ} \mathrm{C}$ ), onde se constata que a devitrificação tem início na superfície, em sítios de baixa energia, por nucleação heterogênea e o crescimento da fase cristalina ocorre, inicialmente ao longo da superfície, em duas dimensões, seguido por um crescimento em direção ao centro da peça. ${ }^{34}$ A Figura $3 \mathrm{c}$ ilustra a interface vidro-cerâmica formado no vidro LNCP após tratamento térmico a $830{ }^{\circ} \mathrm{C}$ e é representativa dos dois tempos de tratamento térmico. Na Figura $3 \mathrm{c}$ estão indicadas as regiões analisadas utilizandose espectroscopia Raman com resolução espacial, ${ }^{16} \mathrm{e}$ os respectivos espectros Raman estão ilustrados na Figura 3d.

No processo de transformação de fase por cristalização com o crescimento controlado por interface, a concentração de soluto inicial na matriz e a concentração próxima da interface permanecem constantes durante o processo de crescimento. ${ }^{35,36} \mathrm{Na}$ Figura $3 \mathrm{c}$, a região (i) apresenta um espectro Raman igual ao do vidro original (Figura 3d-i), confirmando
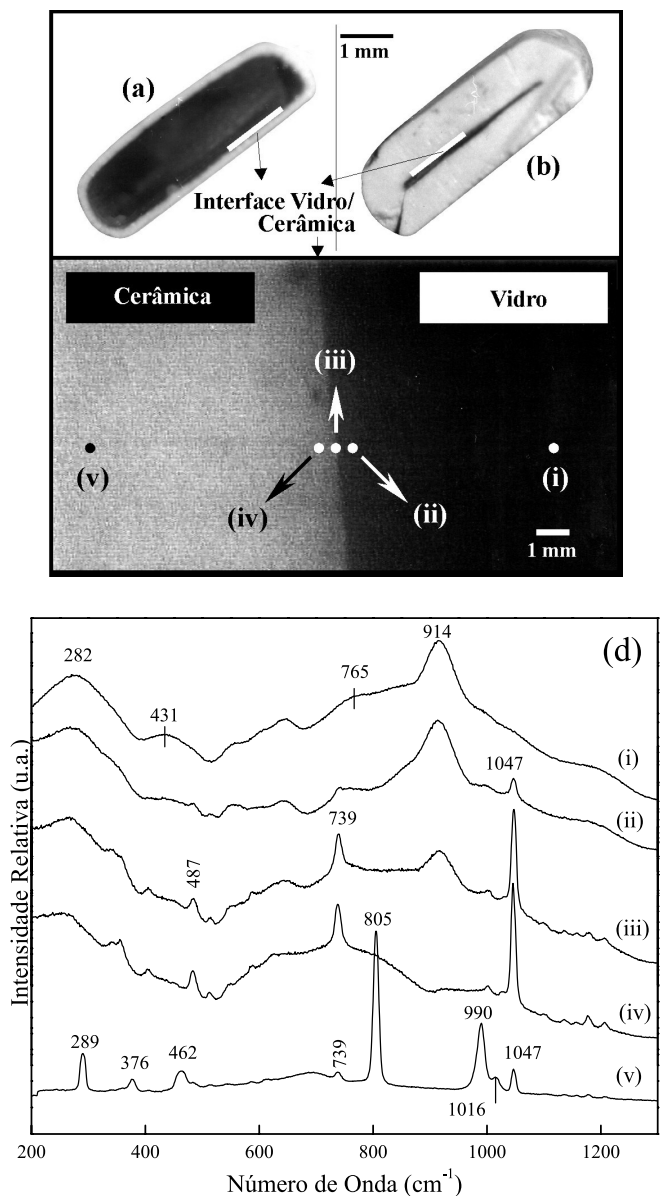

Figura 3. Microscopia óptica do vidro LNCP após (a) 2 min e (b) 5 min de tratamento térmico a $830^{\circ} \mathrm{C}$; (c) interface vidro-cerâmica formado no vidro LNCP representativa dos dois tempos de tratamento térmico a $830^{\circ} \mathrm{C} ;(d)$ espectros Raman das regiões indicadas na Figura 3c. Reproduzida da ref. 16, com permissão da Spinger tratar-se de uma região vítrea. Na região (ii) da Figura 3c, denominada de região nucleada, observamos a ocorrência da banda em $914 \mathrm{~cm}^{-1}$ (típica da ligação $\mathrm{Nb}-\mathrm{O}$ na estrutura vítrea) e uma banda em $1047 \mathrm{~cm}^{-1}$, atribuída ao $\left[\mathrm{V}_{\mathrm{s}}\left(\mathrm{PO}_{3}{ }^{2-}\right)\right]$ da fase $\beta-\mathrm{Ca}_{2} \mathrm{P}_{2} \mathrm{O}_{7}$ (Figura $3 \mathrm{~d}$-ii) ${ }^{31} \mathrm{Na}$ interface, região (iii) da Figura 3c, verificamos uma redução da intensidade da banda em 914 $\mathrm{cm}^{-1}$ e a intensificação da banda em $1047 \mathrm{~cm}^{-1}$, além do surgimento de outras duas bandas: uma em $739 \mathrm{~cm}^{-1}$, atribuída ao $\left[\mathrm{v}_{\mathrm{s}}(\mathrm{POP})\right]$ e outra em $487 \mathrm{~cm}^{-1}$, atribuída a $\left[\delta_{\mathrm{s}}\left(\mathrm{PO}_{3}{ }^{2-}\right)\right]$, ambas também associadas à fase $\beta-\mathrm{Ca}_{2} \mathrm{P}_{2} \mathrm{O}_{7}$ (Figura 3d-iii). Na região (iv) da Figura 3c, correspondente à região cristalizada próxima a interface, não se observa a banda em $914 \mathrm{~cm}^{-1}$, apenas as bandas típicas da fase $\beta-\mathrm{Ca}_{2} \mathrm{P}_{2} \mathrm{O}_{7}$ (Figura 3d-iv). Na região (v), Figura $3 c$, correspondente a uma região cristalizada mais distante da interface, obtém-se um espectro Raman (Figura 3d-v) completamente diferente da região (iv). As bandas em $1016 \mathrm{~cm}^{-1}$ [v(P-O)], $990 \mathrm{~cm}^{-1}$ [v(P-O)], $805 \mathrm{~cm}^{-1}$ [v(Nb-O) curta distância], 462, 376 e 293 $\mathrm{cm}^{-1}$, estas últimas três atribuídas a $[\delta(\mathrm{O}-\mathrm{P}-\mathrm{O})+\delta(\mathrm{O}-\mathrm{Nb}-\mathrm{O})]$, estão associadas aos modos vibracionais da fase $\alpha-\mathrm{NbPO}_{5} \cdot{ }^{37,38}$ Apenas as bandas mais intensas da fase $\beta-\mathrm{Ca}_{2} \mathrm{P}_{2} \mathrm{O}_{7}\left(1047 \mathrm{e} 739 \mathrm{~cm}^{-1}\right)$ manifestam-se como bandas de baixa intensidade no espectro Raman da região (v). Isso ocorre porque a banda do grupo niobila $(\mathrm{Nb}=\mathrm{O}), \mathrm{em} 805 \mathrm{~cm}^{-1}$, é extremamente intensa (Figura 3d-v). Esse resultado mostra que a nucleação tem início com a formação de núcleos da fase $\beta-\mathrm{Ca}_{2} \mathrm{P}_{2} \mathrm{O}_{7}$, os quais induzem uma separação de fases na matriz vítrea, levando à formação de uma segunda fase, formada pelo $\alpha-\mathrm{NbPO}_{5}$.

Com o avanço da frente de cristalização, a concentração da interface permanece constante, sendo portanto, uma evidência de que o crescimento é controlado por um processo de interface.

$\mathrm{O}$ vidro LVCP apresenta processo de transformação de fase semelhante ao apresentado pelo vidro LNCP. A Figura 4a apresenta a interface vidro/cerâmica, que foi analisada utilizando-se espectroscopia Raman com resolução espacial. Os espectros Raman estão ilustrados na Figura 4b.

A interface vidro/cerâmica observada na Figura 4a é similar à obtida para o vidro LNCP após tratamento térmico a $600{ }^{\circ} \mathrm{C}$ por 5 min. O espectro Raman da região (i) na Figura 4 é similar ao reportado para o vidro LVCP original, caracterizado pela banda centrada em $930 \mathrm{~cm}^{-1}$, atribuída às ligações $\mathrm{V}-\mathrm{O}$ e a fosfatos de cadeias curtas na rede do vidro. ${ }^{39} \mathrm{O}$ espectro Raman da região (ii) indica um processo de nucleação de fases cristalinas e a manutenção da rede vítrea evidenciada pela banda em $930 \mathrm{~cm}^{-1}$ (Figura 4b-ii). Os espectros Raman das regiões (iii) e (iv) apresentam duas bandas em 880 e 900 $\mathrm{cm}^{-1}$ atribuídas às fases $\beta-\mathrm{Ca}_{2} \mathrm{~V}_{2} \mathrm{O}_{7}$ (grupos $\left.\mathrm{V}_{2} \mathrm{O}_{7}{ }^{4-}\right)$ e $\beta-\mathrm{Ca}_{3}\left(\mathrm{VO}_{4}\right)_{2}$ (grupos $\mathrm{VO}_{4}^{3-}$ ), respectivamente. ${ }^{40,41} \mathrm{Na}$ região (iii), a banda atribuída aos grupos $\mathrm{V}_{2} \mathrm{O}_{7}^{4-}$ é mais intensa, enquanto na região (iv) a banda atribuída ao grupo $\mathrm{VO}_{4}{ }^{3-}$ é a mais intensa, indicando uma transição de fase (Figuras $4 b$, iii e iv, respectivamente). O espectro Raman da vitrocerâmica obtida após $12 \mathrm{~h}$ de devitrificação do vidro LVCP indica a banda atribuída ao grupo $\mathrm{VO}_{4}^{3-}$ como sendo a mais intensa. Tal evolução estrutural, observada na interface vidro/cerâmica que se estende por alguns micrometros, não poderia ser evidenciada por técnicas cuja amostragem exigisse destruição da amostra (como XRD e FTIR).

\section{CONCLUSÃO}

A partir de medidas de espectroscopia micro-Raman em pontos distintos da superfície de vidros LTCP, LNCP e LVCP, tratados termicamente por diferentes intervalos de tempo nas temperaturas de devitrificação, obtiveram-se evidências que permitiram determinar os mecanismos de devitrificação. Para o vidro LTCP, com o tempo de tratamento térmico, a estrutura vítrea gradualmente se converte nos arranjos ordenados típicos das fases cristalinas $\mathrm{LiTi}_{2}\left(\mathrm{PO}_{4}\right)_{3}$, $\beta-\mathrm{Ca}_{2} \mathrm{P}_{2} \mathrm{O}_{7}$ e $\mathrm{TiO}_{2}$. Posteriormente, a quebra de ligações em ponte 

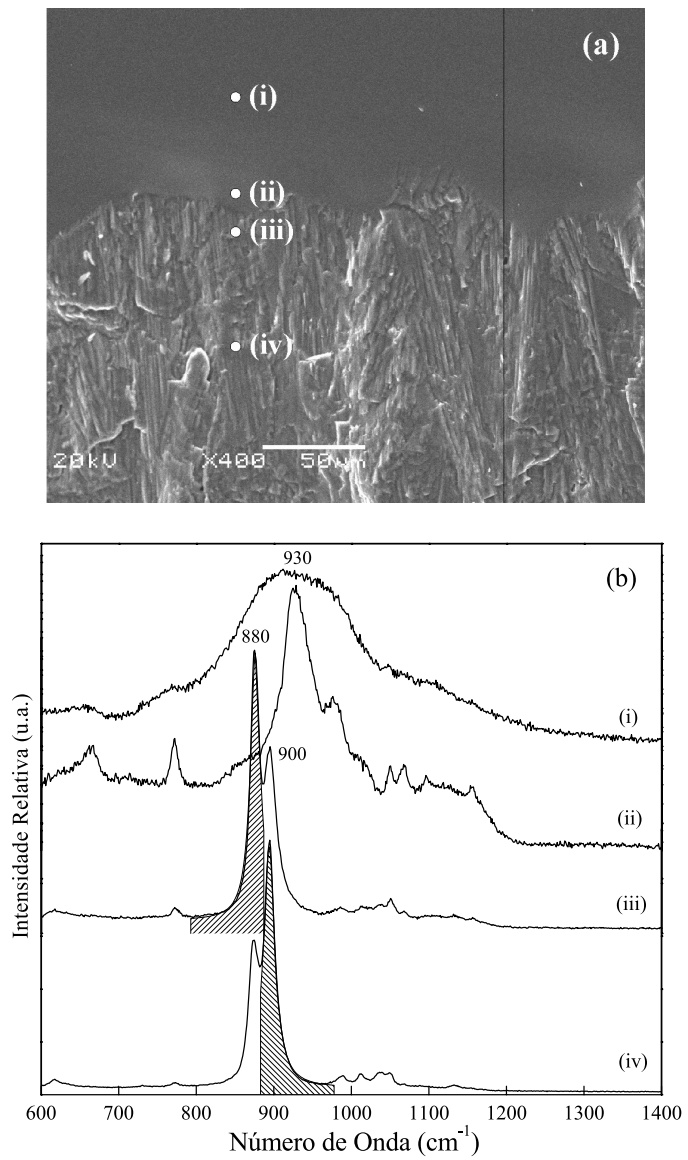

Figura 4. (a) Micrografia SEM da interface vidro/cerâmica obtida após tratamento térmico do $\mathrm{LVCP}$ a $830^{\circ} \mathrm{C}$ por 2 min e (b) espectros Raman das regiões indicadas na Figura 4 a

de cadeias residuais do vidro e da própria fase $\mathrm{LiTi}_{2}\left(\mathrm{PO}_{4}\right)_{3}$ forma as fases $\beta-\mathrm{Ca}_{3}\left(\mathrm{PO}_{4}\right)_{2}$ e $\mathrm{Li}(\mathrm{TiO}) \mathrm{PO}_{4}$. Estas modificações graduais são compatíveis com a separação de fases por decomposição espinoidal, visto que as composições das fases inicialmente separadas variam em função do tempo. Para o vidro LNCP, observa-se uma interface relativamente bem definida entre a região vítrea e a região cristalizada (caracterizada pelas fases $\alpha-\mathrm{NbPO}_{5}$ e $\beta-\mathrm{Ca}_{2} \mathrm{P}_{2} \mathrm{O}_{7}$ ). Nas regiões próximas à interface, observam-se diferenças nas razões de intensidade das bandas atribuídas a estas fases, indicando mudanças nas proporções relativas das fases, porém a composição das mesmas não varia, em função do tempo, com o progresso da devitrificação. Observações semelhantes foram realizadas para o vidro LVCP, de modo que para os vidros LNCP e LVCP o mecanismo proposto é de cristalização controlada por interface.

Tal evolução estrutural, observada na interface vidro/cerâmica que se estende por alguns micrometros, não poderia ser evidenciada por técnicas cuja amostragem exigisse destruição da amostra, evidenciando a potencialidade da espectroscopia micro-Raman como ferramenta analítica para a investigação da variação composicional no processo de transformação de fase em materiais vítreos.

\section{AGRADECIMENTOS}

Ao Laboratório de Espectroscopia Molecular (LEM - IQ/USP) pela possibilidade de utilização do espectrômetro micro-Raman e suporte na realização das medidas, à FAPESP e ao CNPq.

\section{REFERÊNCIAS}

1. Alves, O. L.; Gimenez, I. F.; Mazali, I. O.; Química Nova na Escola, Caderno Temático - Materiais, 2001.

2. Turrell, G.; Corset, J., eds.; Raman Microscopy: Developments and Applications, Academic Press: London, 1996.

3. Anderson, M. E.; Mugli, R. Z.; Anal. Chem. 1981, 53, 1772.

4. Smith, G. D.; Clark, R. J. H.; J. Archaeol. Sci. 2004, 31, 1137.

5. Sala, O.; Quim. Nova 2007, 30, 1773.

6. Sala, O.; Quim. Nova 2007, 30, 2057.

7. Sala, O.; Quim. Nova 2008, 31, 914.

8. Faria, D. L. A.; Souza, M. A.; J. Raman Spectrosc. 1999, 30, 169.

9. Kalasinsky, K. S.; Kalasinsky, V. F.; Spectrochim. Acta A 2005, 61, 1707.

10. Vandenabeele, P.; Castro, K.; Hargreaves, M.; Moens, L.; Madariaga, J. M.; Edwards, H.G. M.; Anal. Chimn. Acta 2007, 588, 108.

11. Maier, M. S.; Faria, D. L. A.; Boschin, M.; Parera, S. D.; Bernal, M. F. D.; Vibrat. Spectrosc. 2007, 44, 182.

12. Faria, D. L. A.; Edwards, H. G. M.; Villar, S .J.; David, A. R.; Anal. Chim. Acta 2004, 503, 223.

13. Colomban, P.; Appl. Phys. A 2004, 79, 167.

14. Gouadec, G.; Colomban, P.; Prog. Crys. Growth Charact. Mater. 2007, $53,1$.

15. Mazali, I. O.; Alves, O. L.; J. Braz. Chem. Soc. 2004, 15, 464.

16. Mazali, I. O.; Alves, O. L.; J. Mater. Sci. Lett. 2001, 20, 2113.

17. Gimenez, I. F.; Mazali, I. O.; Alves, O. L.; J. Phys. Chem. Solids 2001, $62,1251$.

18. Sugita, H.; Tomna, T.; Benino, Y.; Tomatsu, T.; Solid State Commun. 2007, 143, 280 .

19. Koshiba, K.; Tomna, T.; Benino, Y.; Tomatsu, T.; Appl. Phys. A 2007, $89,981$.

20. Avansi, W.; Mastelaro, V. R.; Andreeta, M. R. B.; J. Non-Cryst. Solids 2008, 354, 279 .

21. Strnad, Z.; Glass-Ceramic Materials. Elsevier: Amsterdam, 1986.

22. Mazurin, O. V.; Phase Separation in Glass. Elsevier: Amsterdam, 1984

23. Avrami, M.; J. Chem. Phys. 1939, 7, 1103.

24. Hosono, H.; Abe, Y.; J. Non-Cryst. Solids 1995, 190, 185.

25. Yamamoto, K.; Kasuga, T.; Abe, Y.; J. Am. Ceram. Soc. 1997, 80, 822.

26. Brow, R. K.; Tallant, D. R.; Warren, W. L.; McIntyre, A.; Day, D. E; Phys. Chem. Glasses 1997, 38, 300.

27. Krimi, S.; El Jazouli, A.; Rabardel, L.; Couzi, M.; Mansouri, I.; Le Flem, G.; J. Solid State Chem. 1993, 102, 400.

28. Tarte, P.; Rulmont, A.; Merckaert-Ansay, C.; Spectrochim. Acta 1986, $42 A, 1009$.

29. Barj, M.; Lucazeau, G.; Delmas, C. J.; Solid State Chem. 1992, 100, 141.

30. Mazali, I. O.; Alves, O. L. J. Phys. Chem. Solids 2005, 66, 37.

31. Waal, D.; Hutter, C.; Mater. Res. Bull. 1994, 29, 1129.

32. Bamberger, C. E.; Begun, G.; Cavin, O. B.; J. Solid State Chem. 1988, $73,317$.

33. Aza, P. N.; Santos, C.; Pazo, A.; Aza, S.; Cuscó, R.; Artús, L.; Chem. Mater. 1997, 9, 912.

34. Beall, G. H.; MacDowell, J. F.; Chemtech. 1988, 11, 673.

35. MacMillan, P. W.; Glass-Ceramics, $2^{\text {nd }}$ ed., Academic: London, 1979.

36. Doremus, R. H.; Glass Science, $2^{\text {nd }}$ ed., Wiley: New York, 1994.

37. El Jazouli, A.; Parent, C.; Dance, J. M.; Le Flem, G.; Hagenmuller, P.; J. Solid State Chem. 1988, 74, 377.

38. El Jazouli, A.; Parent, C.; Viala, J. C.; Le Flem, G.; Hagenmuller, P.; J. Solid State Chem. 1988, 74, 433.

39. Vedeanu, N.; Cozar, O.; Ardelean, I.; Magdas, D. A.; Vib. Spectrosc. 2008, 48, 259.

40. Kristallov, L. V.; Fotiev, A. A.; Tsvetkova, M. P.; Russ. J. Inorg. Chem. 1982, 27, 1714.

41. Grzechnik, A.; Chem. Mater. 1998, 10, 1034. 